\title{
Filamentation of Campylobacter in broth cultures
}

\author{
Nacheervan M. Ghaffar, Phillippa L. Connerton and lan F. Connerton* \\ Division of Food Sciences, School of Biosciences, University of Nottingham, Loughborough, UK
}

The transition from rod to filamentous cell morphology has been identified as a response to stressful conditions in many bacterial species and has been ascribed to confer certain survival advantages. Filamentation of Campylobacter jejuni was demonstrated to occur spontaneously on entry in to stationary phase distinguishing it from many other bacteria where a reduction in size is more common. The aim of this study was to investigate the cues that give rise to filamentation of $C$. jejuni and $C$. coli and gain insights into the process. Using minimal medium, augmentation of filamentation occurred and it was observed that this morphological change was wide spread amongst $C$. jejuni strains tested but was not universal in C. coli strains. Filamentation did not appear to be due to release of diffusible molecules, toxic metabolites, or be in response to oxidative stress in the medium. Separated filaments exhibited greater intracellular ATP contents (2.66 to $17.4 \mathrm{fg}$ ) than spiral forms (0.99 to $1.7 \mathrm{fg}$ ) and showed enhanced survival in water at 4 and $37^{\circ} \mathrm{C}$ compared to spiral cells. These observations support the conclusion that the filaments are adapted to survive extra-intestinal environments. Differences in cell morphology and physiology need to be considered in the context of the design of experimental studies and the methods adopted for the isolation of campylobacters from food, clinical, and environmental sources.

Keywords: Campylobacter, filamentation, morphological changes, morphotypes, survival, intracellular ATP

\section{Introduction}

Campylobacter is frequently responsible for foodborne bacterial gastroenteritis worldwide (World Health Organization [WHO], 2013). Campylobacter cells are usually slender, spiral shaped rods measuring $0.2-0.8 \mu \mathrm{m}$ wide and $0.5-5 \mu \mathrm{m}$ in length (Vandamme, 2000) but like many other microorganisms, filamentous forms have been observed under certain circumstances (Griffiths, 1993; Thomas et al., 1999; Apel et al., 2012; Cameron et al., 2012). Filamentation has been identified in many different bacteria and is thought to occur through inhibition of cell division, metabolic changes, or DNA damage which includes the SOS response resulting in the inhibition of septum formation whilst the chromosome is repaired (Justice et al., 2008). It has frequently been associated with stress and starvation conditions during which it may confer survival advantages (Justice et al., 2008). Moreover, it has been suggested that filamentation could represent a programmed response to unfavorable environments that aids the bacterium's survival (Justice et al., 2008) and may enhance virulence (Mulvey et al., 2001; Stackhouse et al., 2012). Alternatively, filamentation may simply occur through an inadvertent loss of control of the normal cell division process. Whichever scenario is true may depend on the species of bacteria and the type of environmental stress encountered. Examples of environmental signals that have been identified to induce filamentation include: starvation, exposure to oxidative stress, 
reduced water activity, the presence of quorum sensing molecules, antibiotics, or host immune effectors (Allison et al., 1992; Jones et al., 1996; Janion, 2001; Miller et al., 2004). Filamentation as a response to sublethal stress has been observed in a number of foodborne bacteria, which have led to concerns that these bacteria may rapidly divide once the growth conditions become permissive to cause spoilage or disease (Jones et al., 2013).

Filamentation in Campylobacter has also been observed in response to mutation of the response regulator RacR and its sensor RacS, which are involved in the heat shock response (Apel et al., 2012), in response to treatment with certain antibiotics such as sitafloxacin (Yabe et al., 2010) and as a general response to hyperosmotic stress (Cameron et al., 2012). Importantly, for Campylobacter jejuni and Helicobacter pylori broth cultures, grown in a microaerobic atmosphere, filamentation occurred spontaneously on entry in to stationary phase (Griffiths, 1993; Fawcett et al., 1999; Thomas et al., 1999; Wright et al., 2009). Here nutrients may become depleted, potentially leading to starvation stress or there may be a buildup of metabolites present in the spent medium but the lack of a specific stress trigger distinguishes filamentation of these two related bacterial genera from other bacteria where stationary phase cells are generally reduced in size (Nyström, 2004). Moreover, elongated cells can be readily identified in scanning electron micrographs of Campylobacter biofilms (for examples see Kalmokoff et al., 2006; Brown et al., 2014) indicating filamentation may occur naturally in situations where biofilms form.

The aim of this study was to investigate the cues that give rise to filamentation of C. jejuni growing in broth cultures. We also aimed to investigate the viability of the individual component cells of the filament using vital staining, determine any strain dependency and any possible differences in the ability of the two different morphotypes to survive unfavorable conditions.

\section{Materials and Methods}

\section{Bacterial Strains}

Campylobacter strains that were used for this study included: HPC5, HF5, (C. jejuni poultry isolates); NCTC11168, NCTC12661 (35925B2), 81-176, PT14, 81116 (C. jejuni reference strains isolated from humans); OR4451C, OR5482C (C. coli poultry isolates), and FB1 (C. coli human isolate). All strains were stored at $-80^{\circ} \mathrm{C}$ in Microbank vials (Pro-Lab Diagnostics, Wirral, UK).

\section{Growth in Liquid Cultures}

Nutrient Broth Number 2 (NB2; Oxoid, Basingstoke, UK) and Mueller Hinton Broth (MH; Oxoid) were prepared according to manufacturer's instructions. MEM (minimum essential medium) without glutamine and phenol red (Catalog number 51200038; Life Technologies Ltd, Paisley, UK), with and without addition of $10 \mathrm{mM}$ sodium pyruvate (Sigma Aldrich, Gillingham, UK), as an energy source were also tested. The Campylobacter inoculum was prepared by making a suspension containing approximately $10^{8} \mathrm{CFU} / \mathrm{ml}$ from an overnight culture grown on blood agar (BA; Oxoid) containing $5 \%(\mathrm{v} / \mathrm{v})$ of defibrinated horse blood (TCS, Buckingham, UK) at $37^{\circ} \mathrm{C}$ incubated under microaerobic conditions (approximately $7 \% \mathrm{O}_{2}$, v/v) obtained by the evacuation/replacement technique (Bolton and Coates, 1983). The replacement gas mix contained (5\% v/v $\mathrm{H}_{2}, 10 \% \mathrm{v} / \mathrm{v}$ $\mathrm{CO}_{2}$, and $85 \% \mathrm{~N}_{2}$ ). To prepare growth curves, $0.1 \mathrm{ml}$ of the Campylobacter inoculum was added to three individual $250 \mathrm{ml}$ conical flasks containing $50 \mathrm{ml}$ of medium and the flasks placed in anaerobic jars (Oxoid) under microaeobic conditions generated as described above. The jars were placed in an orbital shaker and shaken at $100 \mathrm{rpm}$ at $37^{\circ} \mathrm{C}$ with sampling at appropriate intervals. For each time point, an aliquot was serially diluted in maximal recovery diluent (MRD; Oxoid) and the microaerobic atmosphere re-generated. Enumeration of campylobacters was carried out by the Miles and Misra method on Campylobacter blood-free selective agar plates without supplement (CCDA; Oxoid) and incubated microaerobically at $42^{\circ} \mathrm{C}$ for $48 \mathrm{~h}$. The morphology of the cells was examined microscopically over 10 independent fields using bright field, epifluorescence and Gram-stain for each time point. Pre-used NB2 was prepared by carrying out the above procedure with incubation for $48 \mathrm{~h}$ and confirmation of the formation of filamentous cells. The cell growth was removed by centrifugation at $13,000 \mathrm{~g}$ for $15 \mathrm{~min}$, and the supernatant filtered through a $0.2 \mu \mathrm{m}$ filter (cellulose acetate, Sartorius Stedim Biotech, Epsom, UK). The pre-used filter sterilized medium was inoculated as described above to prepare growth curves. To reduce the potential accumulation of free radicals in MEM with pyruvate, the medium was supplemented with $0.15 \% \mathrm{w} / \mathrm{v}$ starch (Sigma Aldrich) prior to sterilization. Statistical differences were assessed by ANOVA from the Excel Data Analysis package (Microsoft Corporation, Redmond, WA, USA).

\section{Fluorescent Cell Staining (Syto9/Propidium lodide)}

Bacterial suspensions $(1 \mathrm{ml})$ were mixed with $1.5 \mu \mathrm{l}$ of Syto 9 (absorption/emission 485/498 $\mathrm{nm}$ ) and $1.5 \mu \mathrm{l}$ propidium iodide (absorption/emission 535/617 nm) from LIVE/DEAD ${ }^{\circledR}$ BacLight $^{T M}$ Bacterial Viability Kit (Life Technologies) and incubated in the dark for $20 \mathrm{~min}$ at room temperature. A $5 \mu \mathrm{l}$ aliquot was applied to the center of a clean glass microscope slide. An $18 \mathrm{~mm}^{2}$ coverslip was placed over the suspension. The slides were examined over $10 \mathrm{~min}$ at a magnification of 1,250 (100×, plan Apo) with an epifluorescence microscope (Labophot; Nikon, Tokyo, Japan) and images captured from 10 independent fields using a vertical mounted digital camera.

\section{Separation of Short Spiral and Filamentous Morphotypes}

In order to separate the short spiral and filamentous forms, three independent biological replicates of C. jejuni 12661 or PT14 were inoculated into $500 \mathrm{ml}$ of sterile MEM with sodium pyruvate, to a final density of approximately $10^{5} \mathrm{CFU} / \mathrm{ml}$. The flasks were incubated in a shaking incubator at $100 \mathrm{rpm}$, at $37^{\circ} \mathrm{C}$ under microaerobic conditions for $30 \mathrm{~h}$. The short spiral cells were separated from filamentous cells by collecting the filtrate from the culture that was passed through a $0.8 \mu \mathrm{m}$ sterile nitrocellulose 
membrane (Sigma-Aldrich). The filtration was repeated with a fresh filter. The filamentous forms were obtained by flushing the first filter with fresh MEM (with pyruvate) and collecting the eluate. The suspensions were examined microscopically and diluted to contain approximately $5 \times 10^{6} \mathrm{CFU} / \mathrm{ml}$ in either sterile water (reverse osmosis) or NB2 to examine their survival characteristics.

\section{Comparison of the Survival of the Short Spiral and Filamentous Morphotypes}

Suspensions of the separated morphotypes in either water or NB2 (prepared as described above) were either incubated at 37 or $4^{\circ} \mathrm{C}$ for $96 \mathrm{~h}$ under microaerobic conditions. Viable counts were performed as described above at $24 \mathrm{~h}$ intervals.

\section{Determination of Intracellular ATP}

The ATP concentrations were measured by luciferase luminescence using a commercial kit according to the manufacturer's instructions (Promega, Southampton, UK). Either suspensions of selected morphotypes (prepared as above) or culture suspensions collected at various time points were centrifuged at $13,0000 \mathrm{~g}$ for $15 \mathrm{~min}$ and the cell pellets washed in $1 \mathrm{ml}$ of TA buffer (20 mM Tris-acetate buffer $\mathrm{pH} 7.75$ ) before re-pelleting. The cell suspensions were lysed with $1 \%$ $(\mathrm{w} / \mathrm{v})$ trichloroacetic acid in TA buffer. The ATP assays were performed by adding $10 \mu \mathrm{l}$ of the cell extract to a polypropylene tube containing $100 \mu \mathrm{l}$ of recombinant luciferase/luciferin reagent, followed by gentle mixing and immediate reading in a pre-blanked luminometer (Turner Designs TD 20e, Promega). The signal was integrated over $10 \mathrm{~s}$ with a $2 \mathrm{~s}$ delay, and reported in relative light units (RLU) that could be converted to ATP concentrations using a pre-prepared standard curve. ATP contents were normalized according to either viable counts or microscopic cell counts or protein content (Bradford reagent; Pierce).

\section{Results}

\section{Confirmation of Filamentation on Entry to Stationary Phase and the Imaging of Filament Component Cells by Vital Staining}

Growth curves and microscopic images of C. jejuni strains HPC5 and HF5 growing in NB2 over $48 \mathrm{~h}$ are shown in Figures 1A-C. These data confirm previous observations that on entry to stationary phase, after $24 \mathrm{~h}$, progressively longer filaments are formed (Griffiths, 1993). The morphological changes coincided with different phases of growth. Exponentially growing cells showed typical short spiral forms $(1.5-2.5 \mu \mathrm{m})$, while mid-stationary phase cells had become elongated. Cell populations in decline phase featured long filaments and the appearance of coccal forms. Vital staining was carried out using a combination of Syto 9, which stains intact cells green and propidium iodide, which only penetrates membrane damaged cells, staining them red. This did not produce the expected result, with many motile and therefore essentially live red-stained cells, appearing in the exponentially growing population. It appears

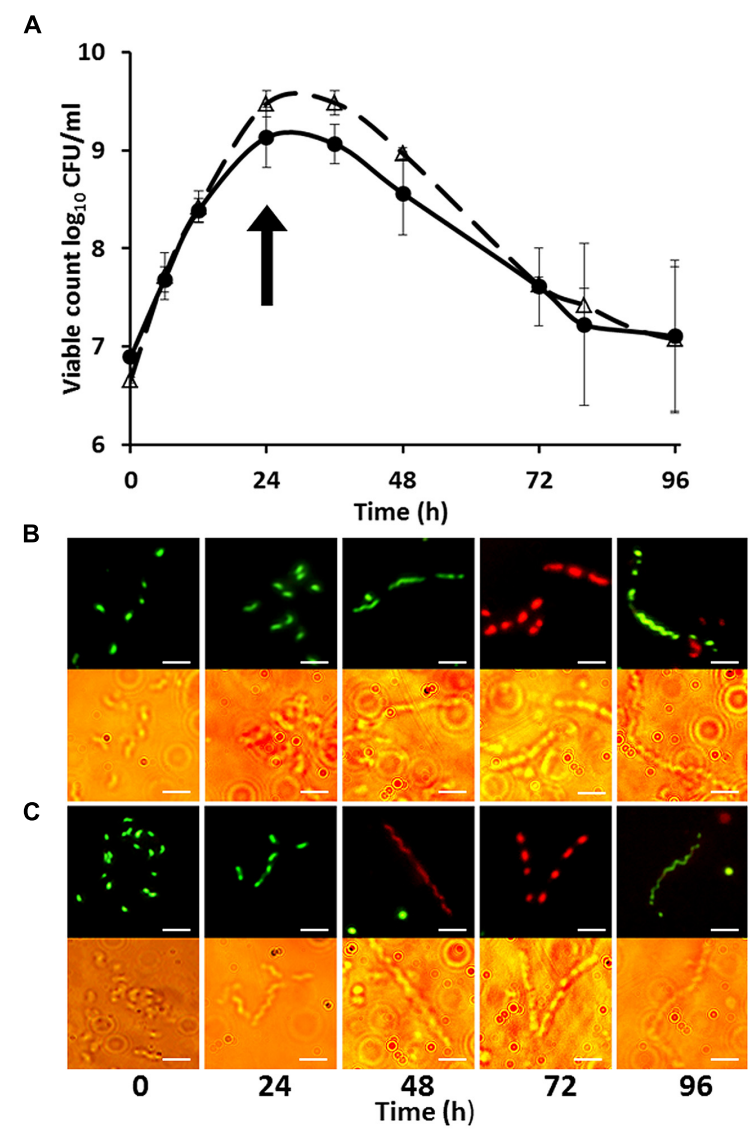

FIGURE 1 | Growth curves (A) of Campylobacter jejuni strains HPC5 $(\triangle)$ and HF5 $(O)$ and morphological changes during growth of $C$. jejuni HPC5 (B), and HF5 (C) in NB2. Arrow in (A) indicates the time point at which the filamentous cells were first observed (on entry to stationary phase). Error bars are $\pm S D$ for $n=3$. In (B) and (C) the upper row shows cells stained with fluorescent stains whilst the lower row shows bright field microscopy image of the same cells. Scale bar, $5 \mu \mathrm{m}$.

that propidium iodide was able to enter live cells and was not a good indicator of viability for Campylobacter. Vital staining of the stationary phase cells showing filamentation was variable, with the cell filaments, either predominantly red (staining with PI) or predominantly green (stained with Syto-9) with relatively few cells containing both fluors (integrated images colored yellow). Interestingly comparison of the bright field and fluorescent microscope images presented in Figures 1B,C, clearly showed that the filaments contained cells that were unstained between the stained cells, and remarkably these often appeared in a regular interspersed pattern as demonstrated at the $72 \mathrm{~h}$ time points for C. jejuni strains HPC5 and HF5 in Figure 1.

\section{Growth of Campylobacter in Pre-Used Medium to Investigate the Potential Role of Quorum Sensing, Depletion of Nutrients}

The exponential growth rates of $C$. jejuni HF5 and HPC5 in the pre-used NB2 medium ( $k=0.14 / \mathrm{h}$ for both strains), were marginally reduced compared to fresh medium $(k=0.18 / \mathrm{h}$ 
for HF5 and $k=0.25 / \mathrm{h}$ for HPC5; Figure 2). This difference was significant for HPC5 $(p=0.03$; ANOVA $)$ but not significant for HF5 ( $p=0.4$; ANOVA). Although not optimum, the pre-used medium was able support exponential growth, suggesting that essential nutrients had not become significantly depleted in the course of achieving stationary phase in the previous culture. Moreover the growth period required for filamentation to occur were similar for fresh and pre-used NB2, suggesting that the campylobacters were not responding to the release of soluble signal molecules as observed for auto inducer molecules involved in quorum sensing.

To investigate further the role of nutritional limitation as a possible cause of filamentation, growth curves were prepared using MEM for C. jejuni HF5 and HPC5. Under these conditions filamentation was observed as early as $2 \mathrm{~h}$ (Figure 2; indicated by arrows), but with no increase in viable count and no filaments greater than $10 \mu \mathrm{m}$ in length (Figures $\mathbf{3 A - C}$ ). Some individual cells were stained both red and green (Figure 3A). Addition of sodium pyruvate, as an energy source, produced an increase in the viable count and filamentation was delayed such that filaments appeared after 4-6 h of incubation. Longer incubation periods produced filaments greater than $10 \mu \mathrm{m}$ in length, which stained with both Syto 9 and PI. This dual staining was
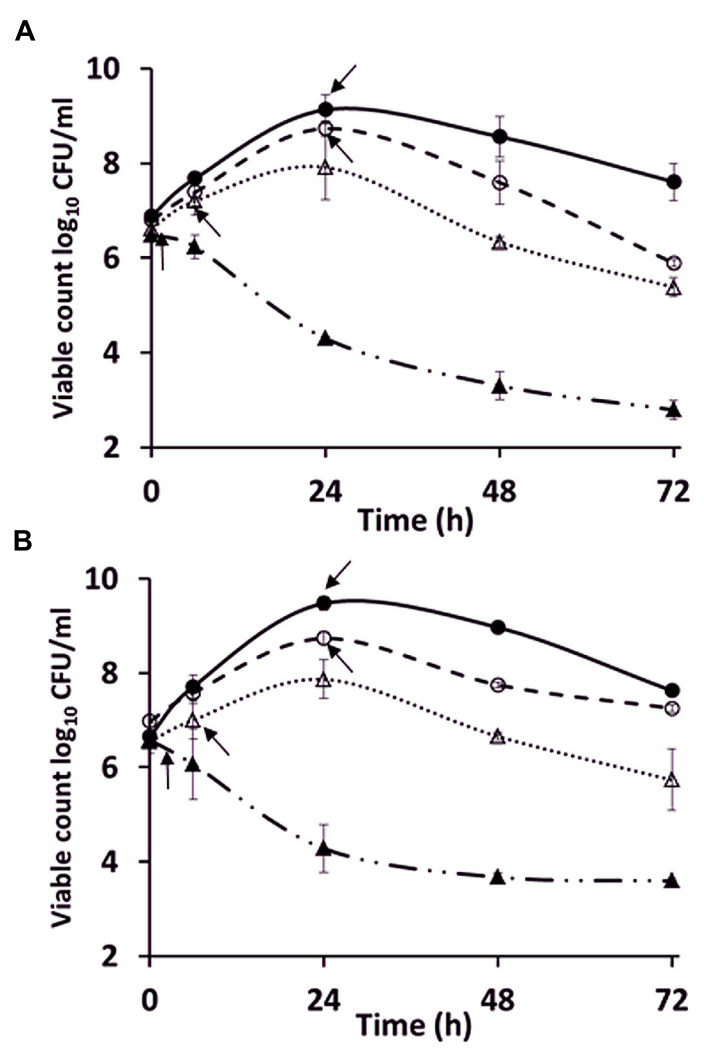

FIGURE 2 | Comparison of the growth of C. jejuni strains (A) HPC5 and (B) HF5 in: NB2 (O), pre-used NB2 (O), minimum essential medium (MEM; $\triangle$ ), and MEM supplemented with $10 \mathrm{mM}$ sodium pyruvate $(\Delta)$. Arrows indicate the time points at which filamentous cells were first observed. Error bars are $\pm \mathrm{SD}$ for $n=3$. particularly evident in a third strain investigated, C. jejuni 12661 (Figures 3D,E).

\section{Growth of Campylobacter in Starch-Containing Media to Reduce Oxidative Stress}

To assess if the provision of starch as an antioxidant, in the culture media could prevent or delay the onset of filamentation, we cultured campylobacters in either Mueller Hinton broth or MEM (with pyruvate) containing starch. Neither of these alternative growth media altered the time of entry to stationary phase or the subsequent observation of filamentous morphotypes (results not shown). The accumulation of growth related oxidative stressors in the culture medium was probably not a causal effect of filamentation.

\section{Filamentation in Different Strains of C. jejuni and C. coli}

To determine if filamention was widespread amongst C. jejuni and $C$. coli a larger group of strains was examined. These were grown in MEM with sodium pyruvate and morphological changes compared in terms of the time taken for filamentation to be first observed (Table 1). Differences were observed, but filamentation was a feature of all the strains tested (cells $>5 \mu \mathrm{m}$ ) apart from C. coli strain (FB1), which did not form filaments, the viable count did not increase, and this strain produced coccal forms as early as $6 \mathrm{~h}$ under these conditions. One strain, C. jejuni 12661, produced particularly long filaments and was therefore chosen for further experiments involving separation of filaments from other morphotypes.

\section{Filamentous Forms Show Increased Survival in Water}

In order to compare the characteristics of the filamentous and spiral cell forms coexisting in stationary phase cultures of C. jejuni 12661 (exaggerated filamentation phenotype) were separated using membrane filtration. A $0.8 \mu \mathrm{m}$ filter prevented the passage of long filamentous forms and did not hinder the passage of short spiral forms present after $30 \mathrm{~h}$ incubation in MEM with sodium pyruvate at $37^{\circ} \mathrm{C}$ under microaerobic conditions. The survival characteristics of the separated morphotypes were examined in both NB2 and in water. There was no significant difference $(p>0.5$; ANOVA) in the ability of the two morphotypes to survive in $\mathrm{NB} 2$ at either 37 or $4^{\circ} \mathrm{C}$ (Figures 4A,B). However, marked differences were observed in the survival of the two morphotypes incubated in water at either temperature (Figures 4C,D). The viable count of the short spiral forms fell below the limit of detection after $72 \mathrm{~h}$ at $37^{\circ} \mathrm{C}$ and $96 \mathrm{~h}$ at $4^{\circ} \mathrm{C}$, whilst the filamentous forms remained detectable at $37^{\circ} \mathrm{C}$ and experienced only a modest fall in viability of $1.5 \log _{10}$ over $96 \mathrm{~h}$ at $4^{\circ} \mathrm{C}$.

\section{ATP Contents of Spiral, Filamentous, and Coccal Cell Types}

In order to study the ATP contents of spiral, filamentous and coccal cell types, C. jejuni 12661 (exaggerated filamentation phenotype) and C. jejuni PT14 (a strain that showed a typical 

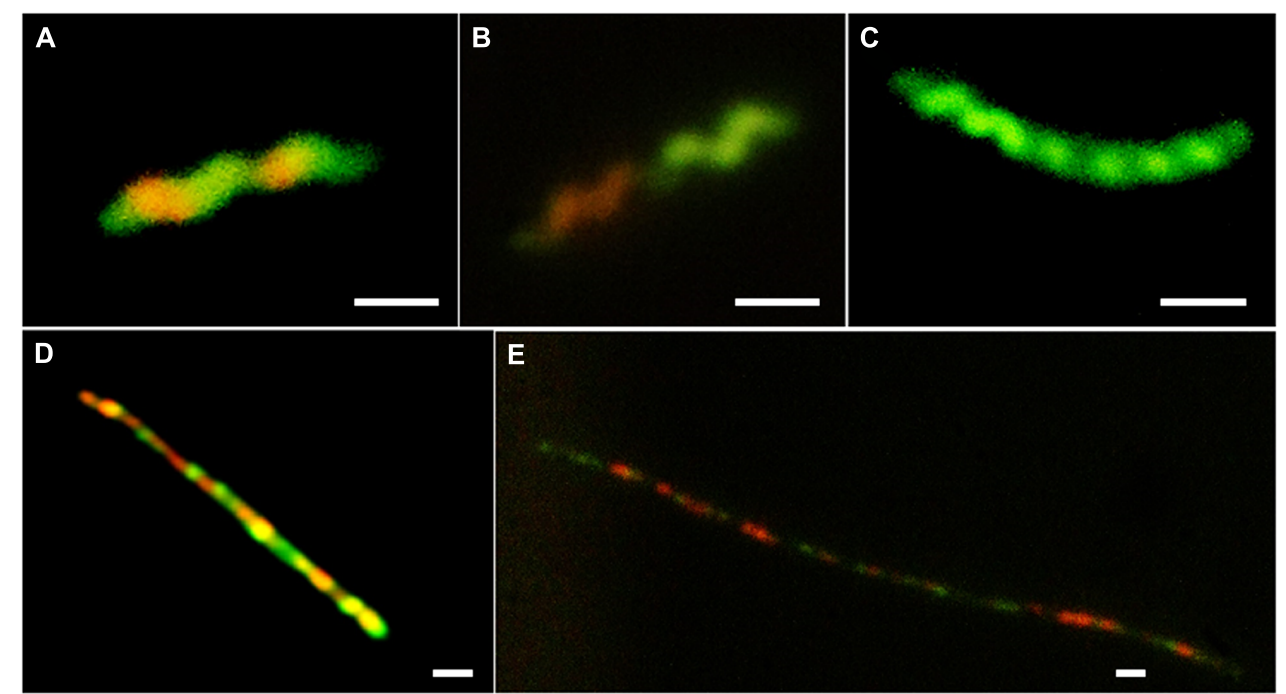

FIGURE 3 | Examples of variation in vital staining observed during growth in MEM and in MEM supplemented with $10 \mathrm{mM}$ sodium pyruvate; (A) C. jejuni HPC5 after $2 \mathrm{~h}$ incubation in MEM,

(B) C. jejuni HPC5 after $4 \mathrm{~h}$ incubation in MEM, (C) C. jejuni HPC5 after $6 \mathrm{~h}$ incubation in MEM; (D) C. jejuni 12661 after $48 \mathrm{~h}$ incubation in MEM supplemented with $10 \mathrm{mM}$ sodium pyruvate; and (E) C. jejuni 12661 after $72 \mathrm{~h}$ incubation in MEM supplemented with $10 \mathrm{mM}$ sodium pyruvate. Scale bar $1 \mu \mathrm{m}$. filamentation phenotype) were selected. Viable and microscopic counts of microaerobic cultures at $37^{\circ} \mathrm{C}$ of C. jejuni 12661 and PT14 in MEM with sodium pyruvate were collected over $216 \mathrm{~h}$ to encompass exponential, stationary and decline phases of growth (Figure 5). Spiral cells harvested in mid-exponential phase at $14 \mathrm{~h}$ were serially diluted and the ATP contents of these cells estimated

TABLE 1 | Viability and filamentation of Campylobacter strains growing in different media.

\begin{tabular}{|c|c|c|c|c|}
\hline Media & Strain & $\begin{array}{l}\text { Time to } \\
\text { achieve } \\
\text { stationary } \\
\text { phase (h)* }\end{array}$ & $\begin{array}{l}\text { Time to } \\
\text { first } \\
\text { filaments } \\
\text { (h) }{ }^{\dagger}\end{array}$ & $\begin{array}{l}\text { Viable count } \\
\text { at first } \\
\text { filamentation } \\
(\mathrm{CFU} / \mathrm{ml})\end{array}$ \\
\hline \multirow[t]{2}{*}{ NB2 } & HPC5 & 30 & 24 & $3.0 \times 10^{9}$ \\
\hline & HF5 & 30 & 24 & $1.3 \times 10^{9}$ \\
\hline \multirow{12}{*}{$\begin{array}{l}\text { Pre-used } \\
\text { filtered NB2 } \\
\text { Minimum } \\
\text { essential } \\
\text { medium (MEM; } \\
\text { (with sodium } \\
\text { pyruvate) }\end{array}$} & HPC5 & 24 & 24 & $2.5 \times 10^{9}$ \\
\hline & HF5 & 24 & 24 & $8.3 \times 10^{8}$ \\
\hline & HPC5 & 24 & 6 & $1.0 \times 10^{7}$ \\
\hline & HF5 & 24 & 6 & $1.6 \times 10^{7}$ \\
\hline & $\mathrm{FB} 1^{\$}$ & 24 & $24^{\ddagger}$ & $4.0 \times 10^{6}$ \\
\hline & OR4451C $\$$ & 24 & 6 & $3.32 \times 10^{7}$ \\
\hline & OR5482C $\$$ & 24 & 6 & $6.0 \times 10^{7}$ \\
\hline & 81116 & 24 & 6 & $6.2 \times 10^{7}$ \\
\hline & 11168 & 24 & 6 & $1.7 \times 10^{7}$ \\
\hline & 12661 & 24 & 6 & $2.1 \times 10^{7}$ \\
\hline & $81-176$ & 24 & 6 & $1.3 \times 10^{7}$ \\
\hline & PT14 & 24 & 6 & $5.2 \times 10^{7}$ \\
\hline \multirow{2}{*}{$\begin{array}{l}\text { MEM (without } \\
\text { sodium } \\
\text { pyruvate) }\end{array}$} & HPC5 & - & 2 & $2.6 \times 10^{6}$ \\
\hline & HF5 & - & 2 & $1.6 \times 10^{6}$ \\
\hline
\end{tabular}

*Determined from growth curve inoculated with $\sim 10^{6} \mathrm{CFU}$.

${ }^{\dagger}$ Determined microscopically, ${ }^{\ddagger}$ Double length cells, ${ }^{\$}$ Campylobacter coli. from luciferase/luciferin luminescence after lysis (Table 2). The ATP contents of the exponential phase spiral cells ( 0.99 and $1.7 \mathrm{fg}$ ATP per CFU) were similar to earlier estimates for C. jejuni (Ng et al., 1985). Recovery and separation of filamentous and spiral forms in decline phase at $96 \mathrm{~h}$ enabled determination of the ATP contents of these cell populations before the appearance of coccal forms. Beyond $168 \mathrm{~h}$ the majority of the cells appeared coccal in these cultures, and estimates of viability required the plating of $0.2 \mathrm{ml}$ culture volumes on multiple blood agar plates and/or recovery at endpoint dilution in broth cultures. Microscopic evaluation of $216 \mathrm{~h}$ cultures revealed that $61 \%$ of the C. jejuni 12661 cells had become coccal compared with $58 \%$ for $C$. jejuni PT14. After correction for the ATP contents of decline phase spiral and filamentous cells present in these cultures (Figure 5), estimates of the ATP contents of the dominant coccal cells were calculated (Table 2). The low or undetectable ATP contents of the coccal cells would support the conclusion that they are no longer viable, and that the viable campylobacters recovered from these cultures represent the remaining spiral and filamentous forms.

\section{Discussion}

Growth curves and microscopic examination of broth grown cultures confirmed that filamentous morphotypes were formed upon entry to stationary phase. Vital staining revealed interesting structural features of the filamentous cells but also that this method may not be suitable for distinguishing live Campylobacter cells from dead ones. The staining patterns observed suggest growth and septa formation occur within the filaments, and that this leads to differential dye permeability preventing dye migration between cells. Moreover, the interspersed pattern 
A

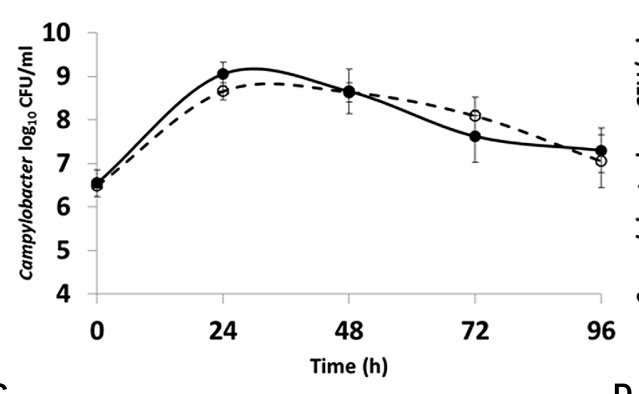

c

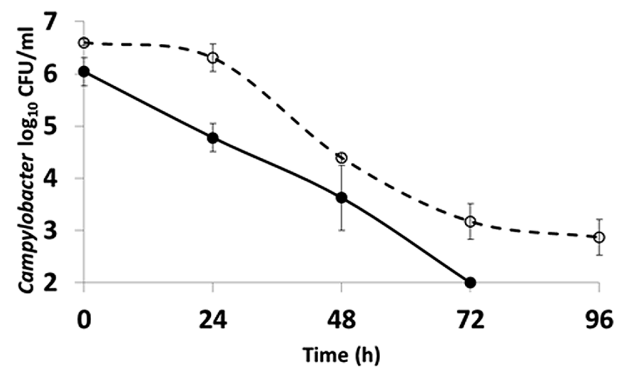

B

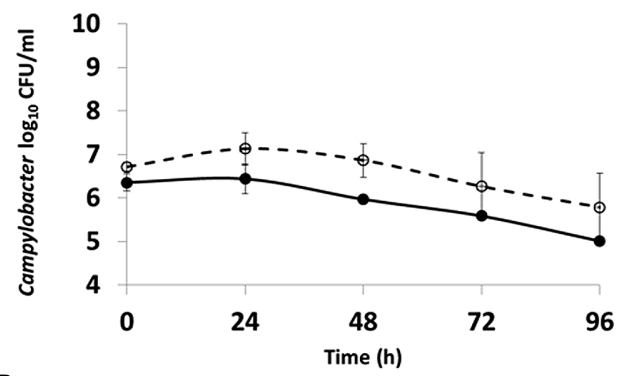

D

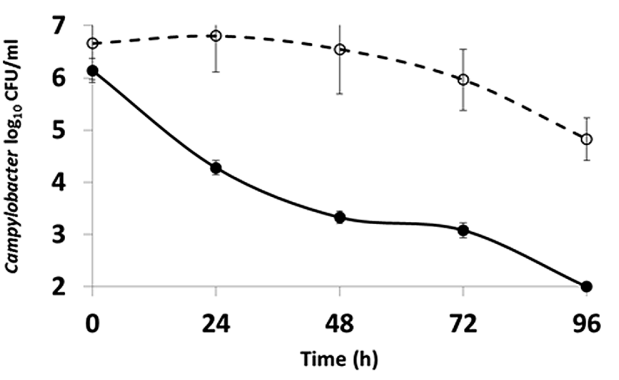

FIGURE 4 | Comparison of the survival of $C$. jejuni 12661 morphotypes incubated microaerobically at: (A) $37^{\circ} \mathrm{C}$ in NB2; (B) $4^{\circ} \mathrm{C}$ in NB2; (C) $37^{\circ} \mathrm{C}$ in water; (D) $4^{\circ} \mathbf{C}$ in water. Short spiral forms $(\mathbf{O})$, filamentous forms (O). Error bars are $\pm S D$ for $n=3$.

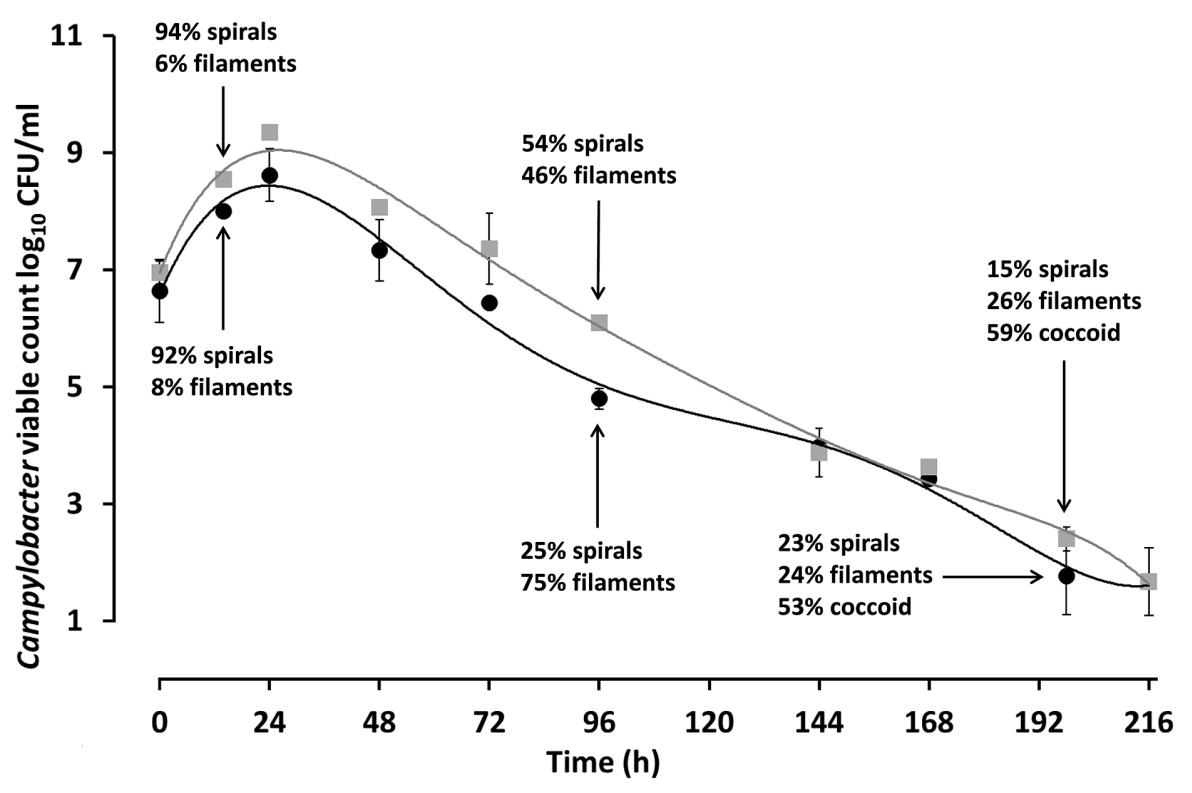

FIGURE 5 | Viable counts and microscopic enumeration of the cell morphotypes observed during microaerobic growth of $C$. jejuni PT14 and 12661. C. jejuni PT14 (-) and $12661(\square)$ were cultured in MEM supplemented with $10 \mathrm{mM}$ sodium pyruvate under microaerobic conditions at $37^{\circ} \mathrm{C}$, from which samples were taken for microscopic examination and estimates of cell bound ATP. Error bars are \pm SD for $n=3$. would suggest cell division may be taking place at the predefined cell poles within the filament suggesting at least some of the component cells were viable. Cameron et al. (2012) observed irregular patterns of cells remaining unstained by PI within Campylobacter filaments formed in response hyperosmotic stress. This prompted the authors to investigate septa formation using Vanco-FL stain that binds D-Ala-D-Ala moieties of peptidoglycan indicative of septa and/or sites of new peptidoglycan synthesis. The Vanco-FL stain produced a punctuated staining pattern in hyperosmotic-induced filaments that did not co-localize with PI. This observation is also indicative of internal septa formation, and as the authors 
TABLE 2 | Intracellular ATP content of $C$. jejuni morphotypes at different phases of growth.

\begin{tabular}{lcc}
\hline Cell Forms* & $\begin{array}{c}\text { C. } \text { jejuni 12661 } \\
\text { ATP (fg/cell) }\end{array}$ & $\begin{array}{c}\boldsymbol{C}^{\dagger} \\
\text { ATP } \text { jejuni PT14 }\end{array}$ \\
\hline Exponential phase spirals (14 h) & $1.7 \pm 0.35$ & $0.99 \pm 0.12$ \\
Decline phase spirals (96 h) & $1.19 \pm 0.20$ & $0.84 \pm 0.14$ \\
Decline phase filaments (96 h) & $17.4 \pm 2.2$ & $2.66 \pm 0.54$ \\
Decline phase coccoid (196 h) & $0.01 \pm 0.005$ & $\mathrm{ND}$ \\
Decline phase coccoid (216 h) & $0.01 \pm 0.004$ & $\mathrm{ND}$ \\
\hline
\end{tabular}

*Cell morphotypes observed at different time points (h) in microaerobic culture of $M E M$ with pyruvate. ${ }^{\dagger} \pm S D n=3$. ND, not detectable.

conclude phenotypic differences between cells that compose the filament.

Quorum sensing bacteria produce and release auto inducers that increase in concentration as a function of cell density leading to an alteration in gene expression (Miller and Bassler, 2001) and in some cases filamentation (Allison et al., 1992). As no change in the time taken to form filaments occurred when pre-used medium was inoculated with a fresh culture, it seems unlikely that quorum sensing was involved in the process. The relatively proficient growth of campylobacters in the pre-used medium was unexpected, as nutrient depletion on entry to stationary phase was suggested as a possible trigger for filamentation. This raises questions as to what actually triggers entry to stationary and decline phases in Campylobacter broth cultures. This is not well understood in other bacteria and probably depends on a combination of factors including the species of bacteria and growth medium. For Salmonella typhimurium and Escherichia coli it has been shown that increased levels of acetate (Wilson et al., 2003) or carbon starvation (Sezonov et al., 2007) are associated with the cessation of growth. The transition to stationary phase for Campylobacter is highly dynamic with a switch from acetate production to utilization together with a peak in motility and numerous gene expression changes (Wright et al., 2009). In addition, the bacteria do not appear to exhibit enhanced stress resistance in stationary phase, unlike many other bacteria, which is consistent with the absence of RpoS homologues (Kelly et al., 2001). Despite the absence of RpoS, campylobacters retain a stringent response that assists survival in the stationary phase of many bacterial species. Mutation of the spoT gene results in aberrant cell morphologies and early coccoid cell formation in stationary phase cultures (Gaynor et al., 2005).

As a microaerophile, Campylobacter is particularly sensitive to the presence of free radicals and may suffer oxidative stress when grown in broth media despite being supplied with a reduced oxygen atmosphere (John et al., 2011). Strictly anaerobic conditions in the presence of alternative electron acceptors nitrate or fumarate have also been shown to induce filamentation in Campylobacter indicating an oxygen requirement for DNA synthesis (Sellars et al., 2002). The presence of starch in Campylobacter culture media acts as an antioxidant and affords a degree of protection against oxidative stress caused by free radicles that can accumulate during exponential growth (Mehlman and Romero, 1982). The provision of starch in the culture media did not influence the timing or degree of filamentation, which suggests that the accumulation of reactive oxygen species (ROS) in the growth medium is not a predisposing factor to the appearance of the filamentation morphotype. However, this does not rule out a role for oxidative stress at a cellular level. Endogenous ROS produced as a consequence of cellular metabolism have been suggested to play a role as signaling molecules and effectors in the development of microbial multi-cellularity, including programed cell death (Čáp et al., 2012).

Campylobacters were unable increase in viable count in MEM, without a carbon source but some increase in cell size was observed. The addition of pyruvate to the MEM allowed growth but with the early onset of filamentation as compared with growth in nutrient rich medium. Nutritional differences clearly impact on the observed morphological changes but since pyruvate remained in excess during the growth period as a carbon and energy source, it is unlikely that filamentation is a response to carbon starvation in these experiments. Bacteria also require sufficient iron, phosphorous, sulfur, nitrogen, and other trace elements for growth and it is possible that one, or a combination of these, become quickly exhausted in minimal medium resulting in filamentation, compared to rich media. However, the response to nutrient limitation even within a well-mixed Campylobacter broth culture is not uniform, in most cultures filamentous types arise among spirals that continue to divide. This implies that once the growth rate has become limited due to nutritional availability, then the formation of the filaments is either a stochastic process or developmentally controlled to generate a subpopulation that are more able to survive nutritional depletion and/or environmental stresses. In the wider environment other limiting physiological factors may also trigger the filamentation response.

Campylobacter jejuni cultured in MEM with pyruvate exhibit an ability to retain a viable subpopulation through decline phase at $37^{\circ} \mathrm{C}$ under microaerobic conditions despite a fall in viable count from $>8 \log _{10} \mathrm{CFU} / \mathrm{ml}$ at the end of exponential phase $\left(24 \mathrm{~h}\right.$ in Figure 5) to $3 \log _{10} \mathrm{CFU} / \mathrm{ml}$ at $168 \mathrm{~h}$. We have measured the ATP contents of cell morphotypes recovered from the decline phases of these cultures to demonstrate significant increases in the cellular ATP contents of the filamentous types as compared with spiral forms, sampled in either exponential phase, or separated from filaments in decline phase. These increases may be accounted for because the filaments appear to consist of multiple cells, joined in an ordered conglomerate. Consistent with this view, is the observation that the C. jejuni strain 12661 produces notably longer filaments and has a higher ATP content (17.4 fg ATP/CFU) than C. jejuni PT14 (2.66 fg ATP/CFU). However, we have noted interspersed staining patterns and cell division within filaments, which could represent cells with greater metabolic activity within a single filament. In the later stages of these cultures ( $>168 \mathrm{~h}$ ) the majority of the cells become coccoid. ATP content estimates of coccal cells were either extremely low or non-detectable, suggesting they are not viable as concluded in previous reports (Moran and Upton, 1986; Boucher et al., 1994). 
Differences in the ability of Campylobacter isolates to survive in microcosm waters have been documented but without reference to the formation of filaments (Buswell et al., 1998). The increased ability of the filamentous morphotype to survive in water compared to the short spiral form suggests that further research is necessary to assess the impact this may have on the transmission of the campylobacters from the environment to farm animals and on the safety of post-process foods. Based on these observations caution is advised when applying mathematical models that predict the survival Campylobacter, but do not take into account that changes in cell morphology and physiology that can increase their probability of survival. The presence of multicellular filaments may also lead to underestimates of viable cell numbers in cultures, since a single filament can form a single colony despite a multicellular origin, and filaments can exhibit differing refraction properties to nondispersed cell suspensions that can make the interpretation of optical density measurements problematic (Wright et al., 2009).

Rapid filamentation was observed in MEM with pyruvate, in all the C. jejuni tested, and in all but one of the C. coli strains tested regardless of the strains being of either poultry or human origin. The response appears widespread amongst the two species. The minimal medium employed in these experiments did not support any observable growth of the single C. coli strain that did not form filaments, it could therefore not be concluded that this strain lacked the capacity to form filaments.

Laboratory based experiments of protein expression and metabolism of Campylobacter demand the use of broth grown cultures to control the phase of growth. Cultures are often harvested in late exponential phase to maximize cell yields,

\section{References}

Allison, C., Coleman, N., Jones, P. L., and Hughes, C. (1992). Ability of Proteus mirabilis to invade human urothelial cells is coupled to motility and swarming differentiation. Infect. Immun. 60, 4740-4746.

Apel, D., Ellermeier, J., Pryjma, M., Dirita, V. J., and Gaynor, E. C. (2012). Characterization of Campylobacter jejuni RacRS reveals roles in the heat shock response, motility, and maintenance of cell length homogeneity. J. Bacteriol. 194, 2342-2354. doi: 10.1128/JB.06041-1

Bolton, F. J., and Coates, D. (1983). Development of a blood-free Campylobacter medium: screening tests on basal media and supplements, and the ability of selected supplements to facilitate aerotolerance. J. Appl. Bacteriol. 54, 115-125. doi: 10.1111/j.1365-2672.1983.tb01308.x

Boucher, S. N., Slater, E. R., Chamberlain, A. H. L., and Adams, M. R. (1994). Production and viability of coccoid forms of Campylobacter jejuni. J. Appl. Bacteriol. 77, 303-307. doi: 10.1111/j.1365-2672.1994.tb03078.x

Brown, H. L., Reuter, M., Salt, L. J., Cross, K. L., Betts, R. P., and van Vliet, A. H. (2014). Chicken juice enhances surface attachment and biofilm formation of Campylobacter jejuni. Appl. Environ. Microbiol. 80, 7053-7060. doi: 10.1128/AEM.02614-4

Buswell, C. M., Herlihy, Y. M., Lawrence, L. M., McGuiggan, J. T., Marsh, P. D., Keevil, C. W., et al. (1998). Extended survival and persistence of Campylobacter spp. in water and aquatic biofilms and their detection by immunofluorescentantibody and -rRNA staining. Appl. Environ. Microbiol. 64, 733-741.

Cameron, A., Frirdich, E., Huynh, S., Parker, C. T., and Gaynor, E. C. (2012). Hyperosmotic stress response of Campylobacter jejuni. J. Bacteriol. 194, 61166130. doi: 10.1128/JB.01409-2 which can contain filamentous forms with potentially different characteristics to exponentially grown short spiral forms. Whilst it has long been recognized that growth in vitro cannot necessarily be compared with growth in vivo, the observation that in vitro grown cells harvested late in the growth cycle show such radically different morphology and survival ability, to cells in exponential phase, requires consideration in the context of experimental design and the methods to be adopted for environmental surveys. The filamentation of Campylobacter strains in laboratory broth culture appears to be a common occurrence. Whether filamentation occurs in all environments inhabited by campylobacters, for example in the intestines of animals and birds, is not known but the presence of filaments in biofilms indicates that this should be further investigated. Similarly there are no reports of filamentous forms observed from environments such as water courses, or from chilled meat, where campylobacters survive but have limited growth potential.

\section{Author Contributions}

NG performed the experimental analyses. NG, PC, and IC designed the experiments, interpreted the data, and wrote the manuscript.

\section{Acknowledgment}

NG was supported by a scholarship from Ministry of Higher Education and Scientific Research, Kurdistan Regional Government, Iraq. Support from the BBSRC of the UK is gratefully acknowledged.

Čáp, M., Vachova, L., and Palkova, Z. (2012). Reactive oxygen species in the signaling and adaptation of multicellular microbial communities. Oxid. Med. Cell Longev. 2012, 976753. doi: 10.1155/2012/976753

Fawcett, P. T., Gibney, K. M., and Vinette, K. M. (1999). Helicobacter pylori can be induced to assume the morphology of Helicobacter heilmannii. J. Clin. Microbiol. 37, 1045-1048.

Gaynor, E. C., Wells, D. H., MacKichan J. K., and Falkow S. (2005). The Campylobacter jejuni stringent response controls specific stress survival and virulence-associated phenotypes. Mol. Microbiol. 56, 8-27. doi: 10.1111/j.13652958.2005.04525.x

Griffiths, P. L. (1993). Morphological changes of Campylobacter jejuni growing in liquid culture. Lett. Appl. Microbiol. 17, 152-155. doi: 10.1111/j.1472765X.1993.tb00382.x

Janion, C. (2001). Some aspects of the SOS response system-a critical survey. Acta. Biochim. Pol. 48, 599-610.

John, A., Connerton, P. L., Cummings, N., and Connerton, I. F. (2011). Profound differences in the transcriptome of Campylobacter jejuni grown in two different, widely used, microaerobic atmospheres. Res. Microbiol. 162, 410-418. doi: 10.1016/j.resmic.2011.02.004

Jones, A. L., Beveridge, T. J., and Woods, D. E. (1996). Intracellular survival of Burkholderia pseudomallei. Infect. Immun. 64, 782-790.

Jones, T. H., Vail, K. M., and McMullen, L. M. (2013). Filament formation by foodborne bacteria under sublethal stress. Int. J. Food Microbiol. 165, 97-110. doi: 10.1016/j.ijfoodmicro.2013.05.001

Justice, S. S., Hunstad, D. A., Cegelski, L., and Hultgren, S. J. (2008). Morphological plasticity as a bacterial survival strategy. Nat. Rev. Microbiol. 6, 162-168. doi: $10.1038 /$ nrmicro1820 
Kalmokoff, M., Lanthier, P., Tremblay, T. L., Foss, M., Lau, P. C., Sanders, G., et al. (2006). Proteomic analysis of Campylobacter jejuni 11168 biofilms reveals a role for the motility complex in biofilm formation. J. Bacteriol. 188, 4312-4320. doi: 10.1128/JB.01975-05

Kelly, A. F., Park, S. F., Bovill, R., and Mackey, B. M. (2001). Survival of Campylobacter jejuni during stationary phase: evidence for the absence of a phenotypic stationary-phase response. Appl. Environ. Microbiol. 67, 2248-2254. doi: 10.1128/AEM.67.5.2248-2254.2001

Mehlman, I. J., and Romero, A. (1982). Improved growth medium for Campylobacter species. Appl. Environ. Microbiol. 43, 615-618.

Miller, C., Thomsen, L. E., Gaggero, C., Mosseri, R., Ingmer, H., and Cohen, S. N. (2004). SOS response induction by beta-lactams and bacterial defense against antibiotic lethality. Science 305, 1629-1631. doi: 10.1126/science.1 101630

Miller, M. B., and Bassler, B. L. (2001). Quorum sensing in bacteria. Annu. Rev. Microbiol. 55, 165-199. doi: 10.1146/annurev.micro.55.1.165

Moran, A. P., and Upton, M. E. (1986). A comparative study of the rod and coccoid forms of Campylobacter jejuni ATCC 29428. J. Appl. Bacteriol. 60, 103-110. doi: 10.1111/j.1365-2672.1986.tb03366.x

Mulvey, M. A., Schilling, J. D., and Hultgren, S. J. (2001). Establishment of a persistent Escherichia coli reservoir during the acute phase of a bladder infection. Infect. Immun. 69, 4572-4579. doi: 10.1128/IAI.69.7.4572-4579.2001

Ng, L. K., Taylor, D. E., and Stiles, M. E. (1985). Estimation of Campylobacter spp. in broth culture by bioluminescence assay of ATP. Appl. Environ. Microbiol. 49, $730-731$.

Nyström, T. (2004). Stationary-phase physiology. Annu. Rev. Microbiol. 58, 161181. doi: 10.1146/annurev.micro.58.030603.123818

Sellars, M. J., Hall, S. J., and Kelly, D. J. (2002). Growth of Campylobacter jejuni supported by respiration of fumarate, nitrate, nitrite, trimethylamine-Noxide, or dimethyl sulfoxide requires oxygen. J. Bacteriol. 184, 4187-4196. doi: 10.1128/JB.184.15.4187-4196.2002

Sezonov, G., Joseleau-Petit, D., and D'Ari, R. (2007). Escherichia coli physiology in Luria-Bertani broth. J. Bacteriol. 189, 8746-8749. doi: 10.1128/JB. 01368-07

Stackhouse, R. R., Faith, N. G., Kaspar, C. W., Czuprynski, C. J., and Wong, A. C. (2012). Survival and virulence of Salmonella enterica serovar enteritidis filaments induced by reduced water activity. Appl. Environ. Microbiol. 78, 2213-2220. doi: 10.1128/AEM.06774-6711

Thomas, C., Hill, D. J., and Mabey, M. (1999). Morphological changes of synchronized Campylobacter jejuni populations during growth in single phase liquid culture. Lett. Appl. Microbiol. 28, 194-198. doi: 10.1046/j.13652672.1999.00504.x

Vandamme, P. (2000). “Taxonomy of the family campylobacteraceae," in Campylobacter, 2nd Edn, eds I. Nachamkin and M. J. Blaser (Washington, DC: American Society for Microbiology), 3-26.

Wilson, P. D., Wilson, D. R., Brocklehurst, T. F., Coleman, H. P., Mitchell, G., Waspe, C. R., et al. (2003). Batch growth of Salmonella typhimurium LT2: stoichiometry and factors leading to cessation of growth. Int. J. Food Microbiol. 89, 195-203. doi: 10.1016/S0168-1605(03)00142-9

World Health Organization [WHO]. (2013). The Global View of Campylobacteriosis Report of an Expert Consultation. Available at: http://www.who.int/foodsafety/publications/foodbornedisease/globalview campylobacterosis/en/

Wright, J. A., Grant, A. J., Hurd, D., Harrison, M., Guccione, E. J., Kelly, D. J., et al. (2009). Metabolite and transcriptome analysis of Campylobacter jejuni in vitro growth reveals a stationary-phase physiological switch. Microbiology 155, 80-94. doi: 10.1099/mic.0.021790-21790

Yabe, S., Higuchi, W., Takano, T., Razvina, O., Iwao, Y., Isobe, H., et al. (2010). In vitro susceptibility to antimicrobial agents and ultrastructural characteristics related to swimming motility and drug action in Campylobacter jejuni and C. coli. J. Infect. Chemother. 16, 174-185. doi: 10.1007/s10156-010-0040-1

Conflict of Interest Statement: The authors declare that the research was conducted in the absence of any commercial or financial relationships that could be construed as a potential conflict of interest.

Copyright (c) 2015 Ghaffar, Connerton and Connerton. This is an open-access article distributed under the terms of the Creative Commons Attribution License (CC BY). The use, distribution or reproduction in other forums is permitted, provided the original author(s) or licensor are credited and that the original publication in this journal is cited, in accordance with accepted academic practice. No use, distribution or reproduction is permitted which does not comply with these terms. 